\title{
Effects of Side-Chain Structure and Solvent on Intramolecular Hydrogen Bonding in Isotactic Poly(methacrylamide)s
}

\author{
Takayuki NakahiRa, ${ }^{\dagger}$ Lin Fan, ${ }^{*}$ Cham Tau Boon, Takayuki FuKada, \\ Takeshi Karato, Masahiko AnNaKa, and Masako YoshikUni \\ Department of Applied Chemistry, Faculty of Engineering, Chiba University, \\ Yavoi-cho 1-33, Inage-ku, Chiba 263-8522, Japan \\ * Graduate School of Science and Technology, Chiba University, \\ Yayoi-cho 1-33, Inage-ku, Chiba 263-8522, Japan
}

(Received March 16, 1998)

\begin{abstract}
Intramolecular hydrogen bonding in isotactic poly(methacrylamide)s, i.e., poly[ $[(S)-1-$ phenylethyl methacrylamide] (1) and isotactic poly[(S)-1-(1-naphthyl)ethyl methacrylamide] (2), was examined by IR, ${ }^{1} \mathrm{H}$ NMR, and CD in non-hydrogen bonding and hydrogen bonding solvents and in mixtures thereof. Isotactic 1 was found to form intramolecular hydrogen bonds more extensively than isotactic $\mathbf{2}$; the former even forms a stable hydrogen-bonded structure in a hydrogen bonding solvent, i.e., dioxane, suggesting that the phenyl groups as well as the methyl groups in the side chains provide adequate steric effects for stabilization of the hydrogen-bonded structure. Formation, in solution, of helical structures of one prevailing handedness with specific chromophore orientation is suggested. The hydrogen-bonded structures were found to break down on addition of a helix-breaking solvent, trifluoroacetic acid.
\end{abstract}

KEY WORDS Poly(methacrylamide) / Intramolecular Hydrogen Bonding / Solvent Effects / Circular

Dichroism

As part of our continuing study of control of side-chain chromophore orientation in polymers to devise "molecular wires" capable of transporting excitation energy and charge one-dimensionally along the polymer chain, ${ }^{1,2}$ we have previously prepared isotactic poly(methacrylamide)s, i.e., poly(1-naphthylmethyl methacrylamide) and poly $[(S)-1-(1-$ naphthyl)ethyl methacrylamide $]$, and showed that they undergo extensive intramolecular hydrogen bonding among the side-chain amide groups in non-hydrogen bonding solvents such as chloroform and 1,2-dichloroethane (EDC) while the corresponding atactic ones do so only partially. ${ }^{3}$ Accordingly, isotactic and atactic poly $[(S)-1-(1-n a p h t h y l) e t h y l$ methacrylamide] were found to give markedly different circular dichroic (CD) spectra in EDC; rather strong Cotton effects observed with isotactic poly $[(S)-1-(1-$ naphthyl)ethyl methacrylamide] suggest the formation of a regular secondary structure, e.g., a one-handed helix, leading to a specific side-chain orientation. A high isotacticity was found to be required for extensive formation of intramolecular hydrogen bonds and hence formation of the regular secondary structure. It was also found that the methyl groups, introduced in the side chains to provide chiral centers in poly $[(S)-1-(1-$ naphthyl)ethyl methacrylamide], have favorable steric effects on the stability of the hydrogen-bonded structure.

In the present study we examined the dependence of intramolecular hydrogen bonding on the solvent and on the side-chain chromophore structure using isotactic poly $[(S)$-1-phenylethyl methacrylamide] (1) as well as isotactic poly [( $S)$-1-(1-naphthyl)ethyl methacrylamide] (2). Isotactic 1 was found to form intramolecular hydrogen bonding even to a larger extent than isotactic 2. We also found that isotactic 1 forms stable intramolecular hydrogen bonds even in a hydrogen bonding solvent, i.e., dioxane, suggesting that the phenyl

† To whom correspondence should be addressed. groups as well as the methyl groups in the side chains provide adequate steric effects to stabilize the hydrogenbonded structure.<smiles>CCC(C)(CC(C)(C)C)C(=O)N[C@@H](C)C(C)Br</smiles>

Ar:<smiles>Cc1ccccc1</smiles>

1

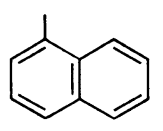

2

\section{EXPERIMENTAL}

\section{Monomeric Model Compounds}

$(S)$-1-Phenylethylacetamide and (S)-1-(1-naphthyl)ethylacetamide and were prepared from the corresponding amines and acetyl chloride and purified in the usual manner.

\section{Polymers}

Isotactic poly(methyl methacrylate) (PMMA) of an isotactic triad content of larger than $99 \%$ and a degree of polymerization of 440 was treated with concentrated sulfuric acid and then with boiling water. ${ }^{4}$ The polymer was shown to be completely hydrolyzed by IR and ${ }^{1} \mathrm{H}$ NMR.

Poly(methacrylamide)s were prepared by condensation of the polymethacrylic acid with amines in dimethylacetamide using dicyclohexylcarbodiimide/1-hydroxybenzotriazole as a condensing agent as reported previously. ${ }^{3}$ The amidation was confirmed to be close to $100 \%$ by elemental analysis and ${ }^{1} \mathrm{H}$ NMR.

\section{Measurements and Calculations}

The IR and ${ }^{1} \mathrm{H}$ NMR spectra were recorded on a JASCO JIR-7000 FT-IR spectrophotometer and on a JEOL GSX-500 ${ }^{1} \mathrm{H}$ NMR spectrometer, respectively. CD 


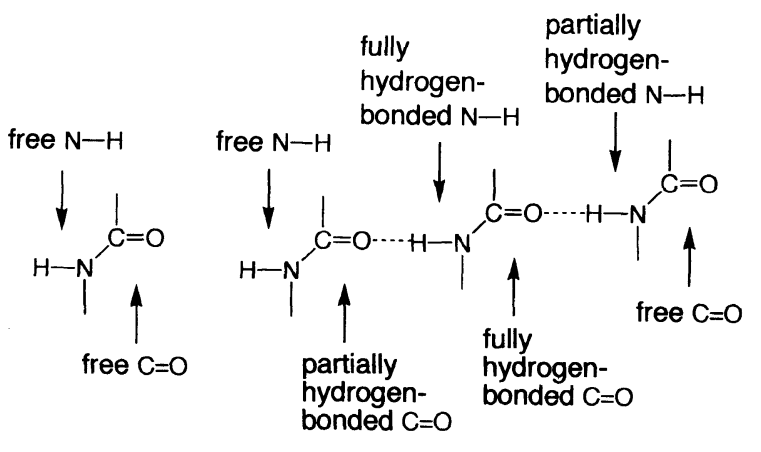

Scheme 1. Hydrogen bonding of side-chain amide groups.

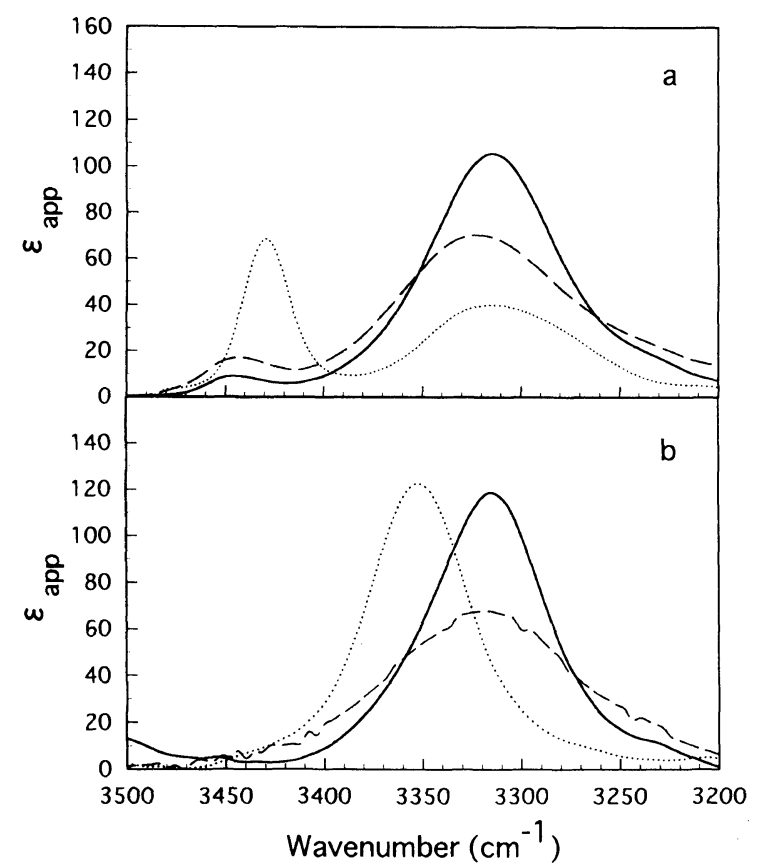

Figure 1. Amide A bands of $0.05 \mathrm{M}$ isotactic 1 (-) and 2 (---) and $0.5 \mathrm{M}(S)$-1-phenylethylacetamide ( $\cdots)$ in EDC (a) and in dioxane (b).

measurements were carried out using a JASCO J-500 circular dichrograph. Wide-angle X-ray diffraction measurements were performed on non-oriented films cast from EDC solutions using a MAC Science MXP X-ray diffractometer with $\mathrm{Cu}-K_{\alpha}$ radiation.

Molecular mechanics calculations were performed with the 24-mers of each polymer using a CAChe molecular mechanics program with an augmented force field for aromaticity. ${ }^{5}$

\section{RESULTS AND DISCUSSION}

As with isotactic 2, isotactic 1 shows extensive hydrogen bond formation in EDC as shown in Figure 1a. As reported previously, ${ }^{3}$ the IR spectra do not change in the concentration range of $0.01-0.5 \mathrm{M}$ (in terms of repeating unit), indicating that the side-chain amide groups are intramolecularly hydrogen-bonded. The relative intensities of amide $\mathrm{N}-\mathrm{H}$ stretching vibration (amide A band) at $c a .3320 \mathrm{~cm}^{-1}$ (fully and partially hydrogen-bonded amide $\mathrm{N}-\mathrm{H}$ ) and at $c a .3440 \mathrm{~cm}^{-1}$ (free amide $\mathrm{N}-\mathrm{H}$ ) show that hydrogen bonding is even more extensive, with a narrower distribution, in isotactic 1 than in isotactic 2. (As previously noted, the broadness of the

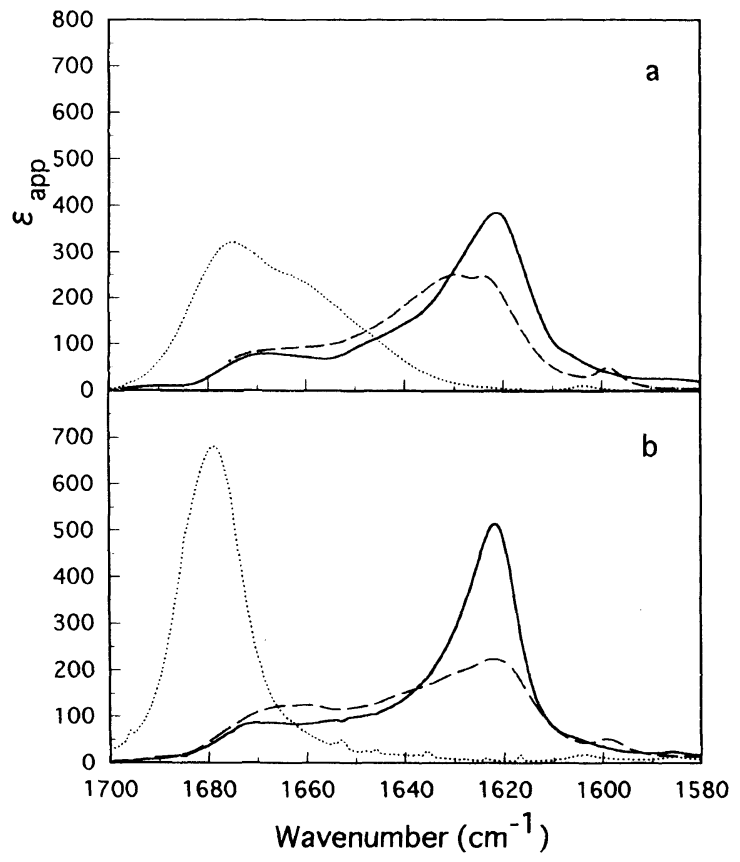

Figure 2. Amide I bands of $0.05 \mathrm{M}$ isotactic 1 (-) and 2 (---) and $0.5 \mathrm{M}(S)$-1-phenylethylacetamide ( $\cdots)$ in EDC (a) and in dioxane (b).

hydrogen-bonded amide $\mathrm{N}-\mathrm{H}$ band reflects, in large part, a distribution of hydrogen-bonded $\mathrm{N}-\mathrm{H}$ groups of varying strength dictated by distance and geometry. ${ }^{6}$ ) The same conclusion is reached when we examine the amide I band region where the absorption of free amide carbonyl appears at $c a .1670 \mathrm{~cm}^{-1}$, that of partially hydrogen-bonded carbonyl at $c a .1640 \mathrm{~cm}^{-1}$, and that of fully hydrogen-bonded carbonyl at $c a .1620 \mathrm{~cm}^{-1}$ (Figure 2a). ${ }^{7}$

In a hydrogen bonding solvent, i.e., dioxane, the model compounds as well as the free amide $\mathrm{N}-\mathrm{H}$ of the polymers are found to be hydrogen-bonded with the solvent (Figure 1b). It is noted that, compared with that in EDC, the amide $\mathrm{A}$ band of isotactic $\mathbf{2}$ in dioxane is slightly broader, suggesting a decrease of fully hydrogen-bonded amide group in dioxane. This is more clearly seen with the amide I band (Figure 2b): an increase of free carbonyl and that of partially hydrogen-bonded carbonyl are noted together with a decrease of fully hydrogenbonded carbonyl. Isotactic 1, on the other hand, maintains virtually all the intramolecular hydrogen bonds that are formed in EDC. In fact, the distribution of fully hydrogen-bonded amide $\mathrm{N}-\mathrm{H}$ as well as that of amide carbonyl appears to be even narrower in dioxane. Thus, the phenyl groups in the side chains, in conjunction with the methyl groups, are suggested to provide better steric effects than the naphthyl groups to stabilize the hydrogen-bonded structure. The ${ }^{1} \mathrm{H}$ NMR spectrum of isotactic $\mathbf{1}$ in chloroform- $d$ and that in dioxane- $d_{8}$ are also virtually identical (Figure 3 ), giving signals at $c a$. 5.8 and $8.4 \mathrm{ppm}$ for free amide protons and for fully hydrogen-bonded amide protons, respectively, in addition to signals at $c a$. 7-7.5 ppm of partially hydrogenbonded amide protons which are buried in the intensive aromatic proton signals. In accord with the above observation, the CD spectrum of isotactic 1 in dioxane is very much similar to that in EDC (Figure 4).

Isotactic 2 in dioxane- $d_{8}$ also gives a ${ }^{1} \mathrm{H}$ NMR 


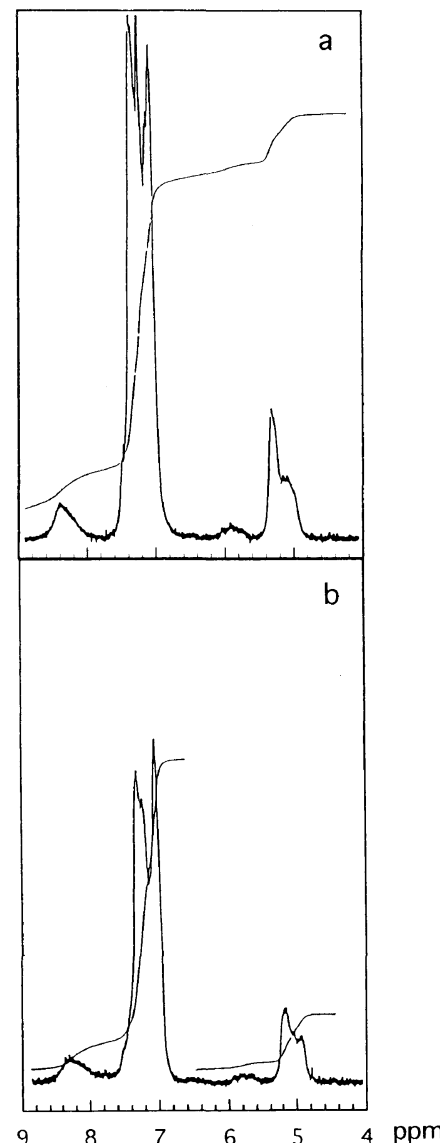

Figure 3. $400 \mathrm{MHz}{ }^{1} \mathrm{H}$ NMR spectra of isotactic 1 in chloroform- $d$ (a) and in dioxane- $d_{8}(\mathrm{~b})$.

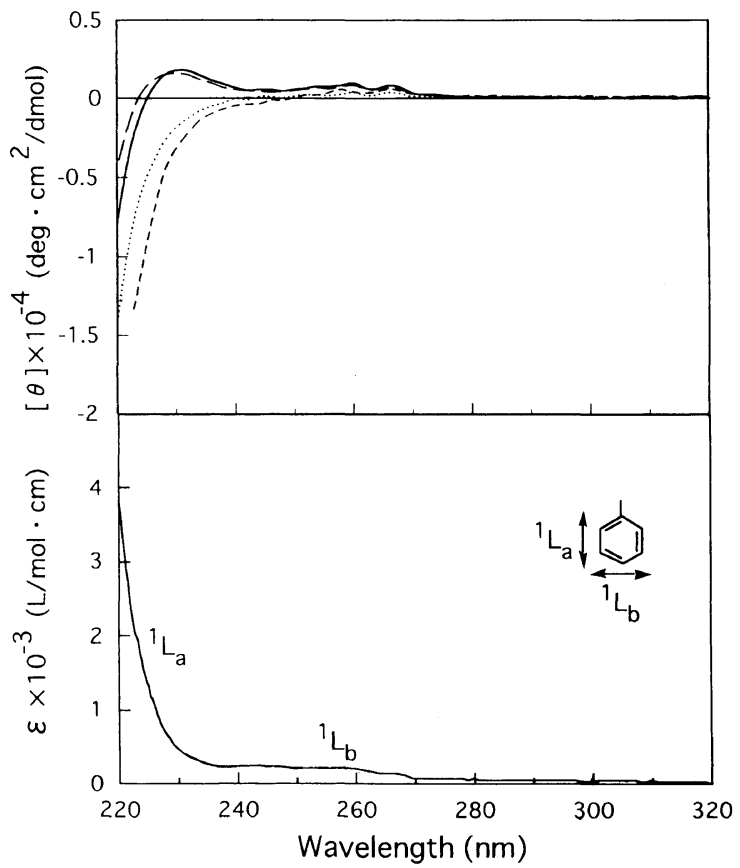

Figure 4. UV and CD spectra of isotactic 1 in EDC (-). Corresponding CD spectra in dioxane (--) and in EDC containing $5 \mathrm{vol} \%$ TFA (--) and that of $(S)$-1-phenylethylacetamide in $\operatorname{EDC}(\cdots)$ are also shown. [Phenyl $]=1 \times 10^{-3} \mathrm{M}$.

spectrum very much similar to that in chloroform-d reported previously. ${ }^{3}$ The CD spectrum of isotactic 2 in dioxane, however, is markedly different from that in

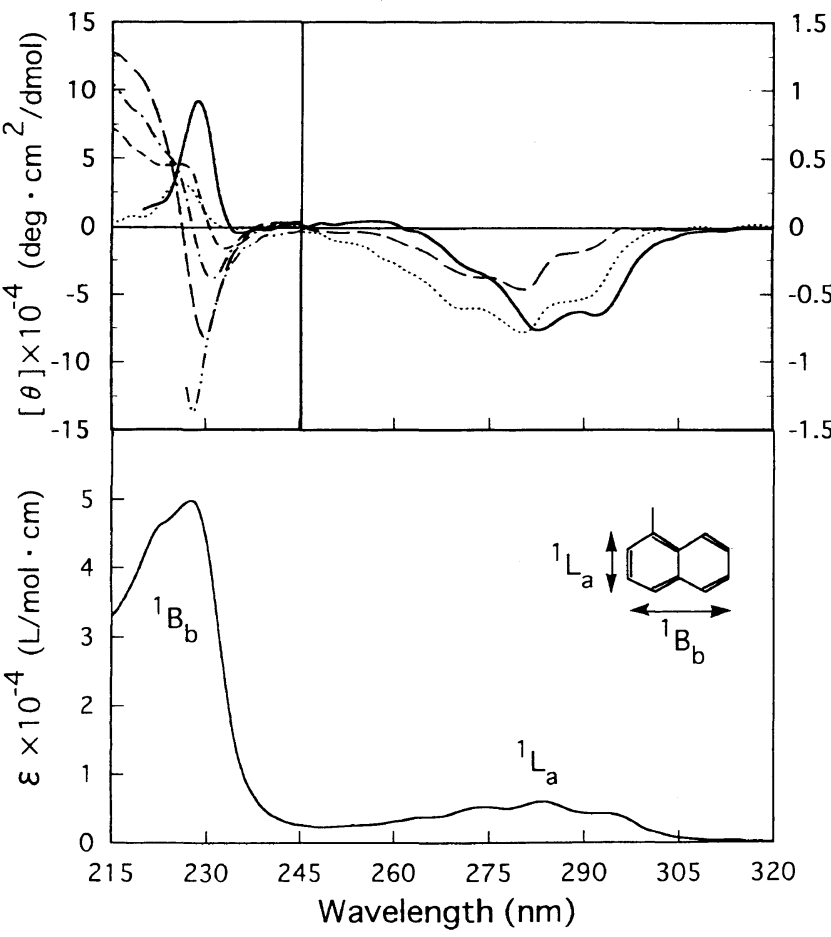

Figure 5. UV and CD spectra of isotactic 2 in EDC (-). Corresponding CD spectra in dioxane (--), in mixed solvents of EDC and dioxane, EDC/dioxane being $1 / 1(---)$ and $3 / 1(--)$, and in EDC containing $5 \mathrm{vol} \%$ of TFA in $225-245 \mathrm{~nm}$ region $(-\ldots--)$ as well as that of $(S)$-1-(1-naphthyl)ethylacetamide in EDC $(\cdots)$ are also shown. $[$ Naphthyl $]=1 \times 10^{-4} \mathrm{M}(215-245 \mathrm{~nm}), 1 \times 10^{-3} \mathrm{M}(245-320 \mathrm{~nm})$.

EDC, apparently reflecting subtle differences, noted by IR, in intramolecular hydrogen bonding in the two solvents (Figure 5). Dependence of CD spectra on the solvent is also reported on a radically polymerized polymethacrylate having pendant L-lactic acid derivatized with trans-4-aminoazobenzene. ${ }^{8}$ Apparently, partial destruction of the "fully hydrogen-bonded structure" by dioxane, which will result in an increase in the relative content of the "partially hydrogen-bonded structure" having free and partially hydrogen-bonded amide groups, strongly affects the overall CD spectrum. The latter structure is suggested to give, in the ${ }^{1} B_{b}$ band of the naphthyl chromophore, an exciton coupling of negative chirality with the first and second Cotton effects being negative and positive, respectively. ${ }^{9}$

When the solvent composition is varied, the CD spectrum of isotactic $\mathbf{2}$ changes continuously (Figure 5). The weak negative peak at ca. $236 \mathrm{~nm}$ observed in EDC suggests that the "partially hydrogen-bonded structure" is present in part in EDC as suggested by IR. We suspect that the "fully hydrogen-bonded structure", when present alone, would give a negative Cotton effect around $220 \mathrm{~nm}$ in addition to the positive Cotton effect at ca. $230 \mathrm{~nm}$, i.e., an exciton coupling of positive exciton chirality in the ${ }^{1} \mathrm{~B}_{\mathrm{b}}$ band of the naphthyl chromophore. (When compared with that of the model compound, the $\mathrm{CD}$ in the ${ }^{1} \mathrm{~L}_{\mathrm{a}}$ band of isotactic $\mathbf{2}$ in EDC is not strictly related to the UV absorption band, suggesting a contribution of exciton coupling of negative exciton chirality, while that in dioxane, though somewhat diminished, suggests a contribution of exciton coupling of positive exciton chirality.) 


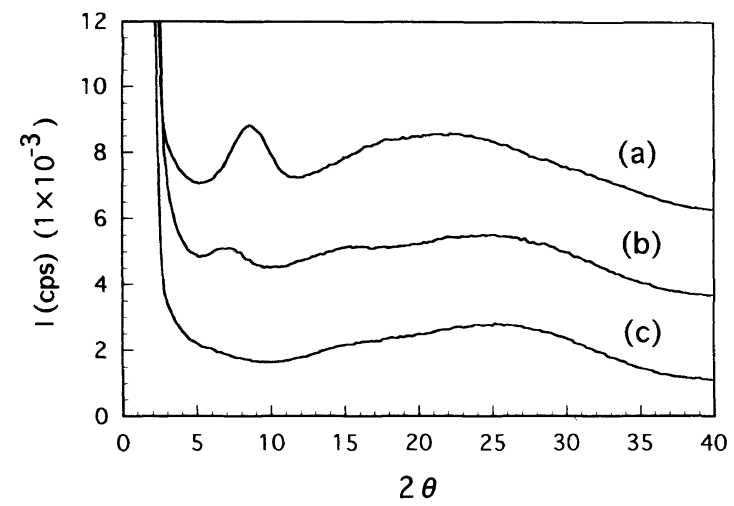

Figure 6. X-Ray diffraction of as-cast films of isotactic 1 (a), isotactic 2 (b), and atactic 2 (c). The films were cast from EDC solutions.

The changes of CD are further demonstrated by adding trifluoroacetic acid (TFA), a known helix-breaking solvent, to the EDC solution of isotactic 2. Addition of $5 \mathrm{vol} \%$ of TFA causes an abrupt change of CD in the ${ }^{1} \mathrm{~B}_{\mathrm{b}}$ band of the naphthyl group, suggesting that the "fully hydrogen-bonded structure" breaks down (Figure 5).

Addition of $5 \mathrm{vol} \%$ of TFA also causes destruction of the "fully hydrogen-bonded structure" of isotactic 1 (Figure 4): the positive peak at ca. $230 \mathrm{~nm}$ observed in EDC as well as in dioxane disappears. We tentatively assign the peak at $c a .230 \mathrm{~nm}$ of isotactic 1 to the $n-\pi^{*}$ absorption band of the side-chain amide groups in the "fully hydrogen-bonded structure". ${ }^{10}$ (We also observed a peak at ca. $230 \mathrm{~nm}$ in the CD spectrum of isotactic poly $[(S)$-1-cyclohexylethyl methacrylamide] in EDC, where no aromatic chromophores are present in the side chains. ${ }^{11}$ ) The corresponding peak for isotactic 2 in EDC appears to be buried in the large CD signal of the ${ }^{1} B_{b}$ band of the naphthyl group.

A question that remains is whether or not the "fully hydrogen-bonded structure" is a helical structure. The X-ray diffraction of the films prepared from isotactic 1 and $\mathbf{2}$ by solvent casting gives peaks other than that in the amorphous region (Figure 6), suggesting that these isotactic polymers are rod-like and, at least in part, pack themselves side by side as is the case with helical polypeptides. $^{12}$

Molecular mechanics calculations show that the isotactic polymethacrylamides can form $\mathrm{ca} .5_{2}$ helices with a pitch of $c a$. $5 \AA$, similar to that suggested for isotactic poly(methyl methacrylate) in crystalline state, ${ }^{13}$ full hydrogen bonding occurring among every other monomer unit, i.e., $i$ and $i+2$, along the polymer chain. No such helices are allowed for syndiotactic sequences.

Calculations on $100 \%$ isotactic model polymers for isotactic $\mathbf{1}$ and $\mathbf{2}$ suggest that there is a significant energy difference between the right-handed and left-handed helices, the right-handed one being more stable by $c a$. $1.7 \mathrm{kcal} \mathrm{mol}^{-1}$ of repeating unit (Figure 7). We thus presume that the right-handed helical structure is likely to be a major secondary structure, giving the observed exciton coupling between the mutually oriented sidechain chromophores.

Another question is: which section of the "partially hydrogen-bonded structure", formed in isotactic 2 (in dioxane) and in atactic $\mathbf{2}$ (both in EDC and in dioxane), ${ }^{3}$ gives an exciton coupling interaction whose chirality in
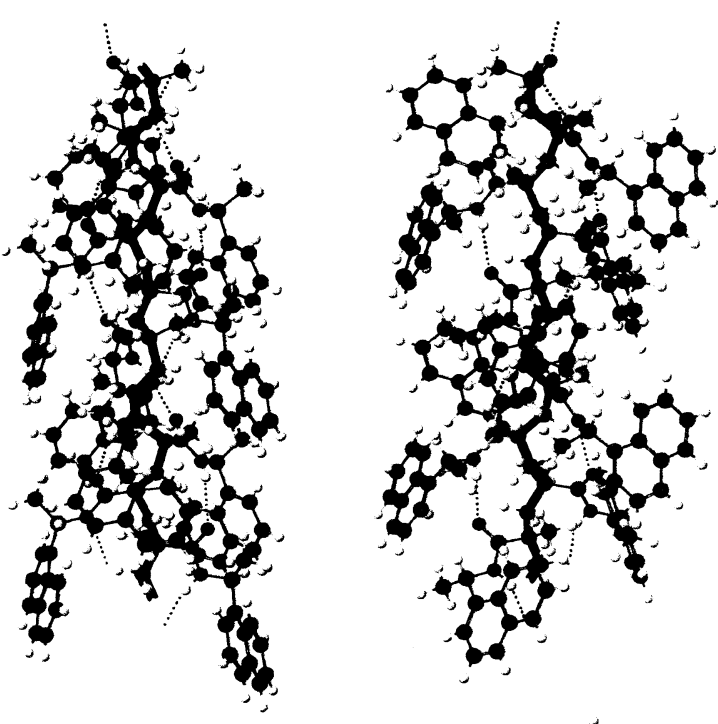

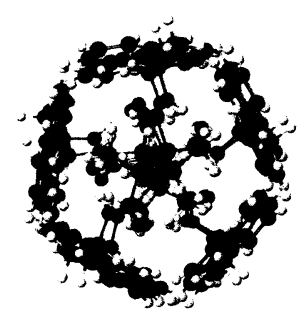

(a)

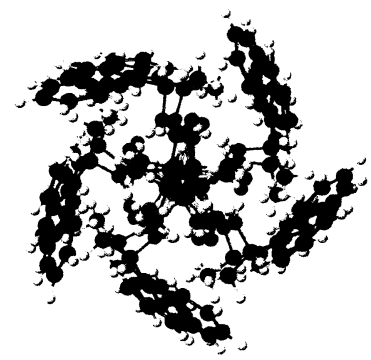

(b)
Figure 7. Ball and stick molecular models for right-handed (a) and left-handed (b) helices of isotactic 2. Dotted lines indicate hydrogen bonding. The calculated conformational energies for the 24-mers of (a) and (b) are -304.5 and $-263.3 \mathrm{kcal} \mathrm{mol}^{-1}$, respectively.

the ${ }^{1} \mathbf{B}_{\mathrm{b}}$ band of the naphthyl chromophore is apparently opposite to that of the "fully hydrogen-bonded structure"? We speculate that the observed exciton coupling is due to two monomer units hydrogen-bonded only to each other, i.e., "dimers", and that these "dimers" are formed between the same $i$ and $i+2$ monomer units along the polymer chain as in the "fully hydrogen-bonded structure" with the intervening $i+1$ monomer unit not hydrogen-bonded to nearby monomer units. They appear to be formed where formation of the "fully hydrogenbonded structure" is not possible, i.e., in EDC in sequences containing syndiotactic junctions and in dioxane in all sequences regardless of tacticity due to hydrogen-bonding solvation of nearby amide groups. Molecular mechanics calculations on such "dimer" models suggest that the mutual orientation of the chromophores can be significantly different from that in the "fully hydrogen-bonded structure". However, we can not exclude the possibility that the observed exciton coupling is due to the section of the "partially hydrogen-bonded structure" which has no intramolecular hydrogen bonding, but still allows side-chain chromophores to take a specific mutual orientation.

\section{CONCLUSION}

Isotactic 1, when compared with isotactic 2, forms stable intramolecular hydrogen bonds among the sidechain amide groups due to the adequate steric effects provided by the phenyl and methyl groups in the side 
chains and maintains the hydrogen-bonded structure even in a hydrogen bonding solvent, i.e., dioxane. The $\mathrm{CD}$ and other spectral data, together with molecular mechanics calculations, suggest that isotactic $\mathbf{1}$ and $\mathbf{2}$ form, at least in part, helical structures of one prevailing handedness with a specific chromophore orientation. These hydrogen-bonded structures break down when helix-breaking TFA is added.

Acknowledgment. This work was supported in part by a Grant-in-Aid for Scientific Research (Grant No. 08651045) from the Ministry of Education, Science, Sports and Culture of Japan.

\section{REFERENCES}

1. T. Nakahira, T. Goto, K. Deguchi, and S. Iwabuchi, $J$. Photopolym. Sci. Tech., 7, 99 (1994).

2. M. Sato, M. Yoshimoto, T. Nakahira, S. Iwabuchi, Makromol. Chem., Rapid Commun., 14, 179 (1993).
3. T. Nakahira, F. Lin, C. T. Boon, T. Karato, M. Annaka, M. Yoshikuni, and S. Iwabuchi, Polym. J., 29, 701 (1997).

4. J. Semen, J . B. Lando, Macromolecules, 2, 570 (1969).

5. S.-Y. Liu and G. D. Purvis, III, "CAChe Molecular Mechanics Augmented Force Field," Tektronix Inc., Beaverton, Oregon, 1991.

6. D. J. Skrovanek, S. E. Howe, P. C. Painter, and M. M. Coleman, Macromolecules, 18, 1676 (1985).

7. N. Tanaka, K. Ito, H. Kitano, Macromolecules, 27, 540 (1994).

8. L. Angiolini, D. Caretti, C. Carlini, and E. Salatelli, Macromol. Chem. Phys., 196, 2737 (1995).

9. K. Nakanishi and N. Berova, in "Circular Dichroism," $K$. Nakanishi, N. Berova, and R. W. Woody, Ed., VCH Publishers, New York, N.Y., 1994, p 361.

10. F. Sanda, M. Nakamura, T. Endo, T. Takata, and H. Handa, Macromolecules, 27, 7928 (1994).

11. L. Fan, T. Fukada, C. T. Boon, T. Karato, M. Annaka, M. Yoshikuni, and T. Nakahira, to be submitted.

12. J. Watanabe, K. Imai, R. Gehani, and I. Uematsu, J. Polym. Sci., Polym. Phys. Ed., 19, 653 (1981).

13. J. D. Stroupe and R. E. Hughes, J. Amer. Chem. Soc., 80, 2341 (1958). 